\title{
Abordaje obstétrico integral de la prematurez y restricción del crecimiento fetal y su relación con las enfermedades crónicas del adulto
}

\author{
Leonel Briozzo*, Stephanie Viroga ${ }^{\dagger}$
}

\section{Resumen}

Los síndromes obstétricos de parto de pretérmino (PP) y restricción de crecimiento fetal (RCIU) comparten mecanismos etiopatogénicos y fisiopatológicos que muchas veces interactúan y se retroalimentan. Desde el punto de vista etiológico, las condiciones específicas que los generan esquemáticamente se pueden clasificar en inflamación, estrés materno, déficit en las condiciones socioeconómicas y vulnerabilidad de derechos, actuación de disruptores endocrinos, alteraciones de la dieta y de la microbiota y afecciones vasculares. Estas condiciones, actuando aislada o más frecuentemente combinadamente, generan un entorno materno desfavorable para el desarrollo del embarazo provocando efectos específicos como son la reacción inmune materna, mediada o no por la presencia de infecciones, la activación del eje hipotálamo-hipófiso-adrenal, la disminución de la acción de la progesterona, las disbiosis, tanto intestinales como vaginales, y la disfunción por envejecimiento placentario. El entorno desfavorable así generado impactará en la unidad útero-placento-fetal, produciendo, bien el PP o bien el RCIU, de acuerdo a la primacía de diferentes respuestas; independientemente de cuál de las respuestas sindromáticas predomine, ambos, el PP y el RCIU, tienen en común el desarrollo del feno-genotipo ahorrador, imprescindible para la sobrevida fetal. El costo de esta modulación epigenética es el aumento de las enfermedades crónicas del adulto, que conceptualmente son enfermedades transmisibles por la vulnerabilidad social donde se desarrolla el ciclo de vida de esas personas.

Palabras clave: Retardo del crecimiento fetal Nacimiento prematuro Enfermedades crónicas Epigenómica

Key words: $\quad$ Fetal growth retardation

Premature birth

Chronic diseases

Epigenomics

* Profesor Titular Clínica Ginecotocológica A.

† Profesora Adjunta Clínica Ginecotocológica A.

Clínica Ginecotocológica A, Centro Hospitalario Pereira Rossell,

Facultad de Medicina, Universidad de la República. Uruguay

Correspondencia: Dra. Stephanie Viroga. Manuel Pagola 3280, Apto. 001, Montevideo, Uruguay. Correo electrónico: stephyviroga@gmail.com

Los autores declaran no tener conflicto de interés con el tema.

Recibido: $25 / 9 / 19$

Aprobado: 4/11/19 


\section{Introducción}

El proceso reproductivo, tanto para la madre como para el feto, es más riesgoso en las mujeres vulneradas en sus derechos. Los dos principales síndromes obstétricos, el síndrome de parto de pretérmino (PP) y el síndrome de restricción de crecimiento fetal (RCIU) son más prevalentes en los sectores vulnerados en sus derechos, tanto a nivel de países subdesarrollados, comparado a los desarrollados, como a lo interno de los países de acuerdo al quintil de ingresos ${ }^{(1,2)}$. Desde el punto fisiopatológico existe una matriz común de condiciones predisponentes de ambos síndromes que repercuten en la salud integral de la mujer embarazada generando efectos patológicos y repercusiones en el complejo útero ovular, con muchas manifestaciones clínicas y complicaciones, también comunes entre ambos síndromes, por lo cual corresponde explorar la existencia de un modelo de abordaje integral de ambos síndromes . A su vez, desde hace ya tiempo, se relacionan la existencia del PP y el RCIU con un riesgo incremental de desarrollar enfermedades crónicas durante la vida adulta, relacionadas fundamentalmente con el síndrome metabólico ${ }^{(3)}$.

\section{Modelo de abordaje integral de la prematurez y la restricción del crecimiento fetal}

Se plantea un modelo con dos niveles de afección, un primer nivel donde un conjunto de condiciones lleva a un entorno materno desfavorable, y, en un segundo nivel, un entorno del complejo útero fetal desfavorable para el desarrollo fetal que puede provocar de acuerdo al peso relativo de cada condicionante prematurez o restricción de crecimiento. En ambos niveles de afección, por mecanismos diversos, algunos no bien conocidos aún, se producen activación epigenética a nivel fetal que llevará a la activación del feno-genotipo ahorrador. Esto se muestra en el mapa conceptual de la figura 1.

\section{Primer nivel: entorno materno desfavorable}

Se describen a nivel materno diferentes condiciones desfavorables que actúan aisladamente o conjuntamente entre sí.

a) Las condiciones inflamatorias, que pueden ser infecciosas o no infecciosas, y provocan una reacción inmune materna distinta de la reacción fisiológica para el desarrollo de un embarazo normal. La infección activa el sistema de receptores tipo Toll 4 (TLR4) trofoblástica que se incrementa por la disminución de la acción de la progesterona, se activa así la cascada de las citoquinas con incremento de TNF, IL6, IL8, provocando una reacción local con aumento de la fosfolipasa A2, las colagenasas, las elastasas, el aumento de la acción de las prostaglandinas, entre otros mecanismos $^{(4)}$.

b) Las condiciones causadas por el estrés materno, como ansiedad y violencia contra la mujer, también se describen como entorno materno desfavorable. El estrés es una reacción fisiológica normal que se da ante circunstancias físicas y fisiológicas que son interpretadas por el individuo como amenazas o de demanda incrementada. Pero, cuando estas amenazas sobrepasan la capacidad adaptativa del estrés se genera una reacción que incrementa el riesgo de que se produzca un daño. El estrés provoca un aumento del cortisol endógeno materno que puede superar la enzima placentaria encargada de su inactivación y llegar así al feto determinando cambios en la metilación del ácido desoxirribonucleico (ADN) fetal ${ }^{(5)}$.

c) Hay evidencias de que la pérdida de la biodiversidad de la microbiota es un factor de riesgo para el desarrollo de enfermedades. La relación entre células microbianas y humanas es entre $2: 1$, se conforma así un ecosistema humano-microbiano que se ha denominado holobionte. Dentro de los factores que modifican la microbiota se encuentra la alimentación, tanto por la disponibilidad de calorías como por la mala calidad de los ultraprocesados. El acceso a determinados tipos de alimentos ultraprocesados con conservantes, la sobreexposición a regímenes calóricos o, por el contrario, la desnutrición, llevan a trastornos como la obesidad o el adelgazamiento extremo, que se relacionan con la generación de alteraciones graves de la microbiota que tendrán efecto deletéreo en la salud de la mujer embarazada y en el proceso reproductivo. Debemos recordar que vivimos en una era de uso indiscriminado de antibióticos que también modifica nuestra microbiota ${ }^{(6)}$.

d) Además se describen las condiciones provenientes de la presencia de disruptores endocrinos vinculados a BETX (benzeno, etil benzeno, tolueno, xileno) y los fenoles. Su uso a nivel del medioambiente ha demostrado efecto sobre la metilación del ADN; por ejemplo, aquellas embarazadas que vivían a menos de $5 \mathrm{~km}$ de emisores de algunas de estas sustancias, presentaron cinco veces más riesgo de parto pretérmino. También se demostró el impacto ambiental mediante la asociación de prematurez con el consumo de ftalatos (sustancias añadidas a plásticos) ${ }^{(7,8)}$

e) Las condiciones de causas vasculares útero placentarias se vinculan de manera directa con la disfunción placentaria. El ejemplo más claro es la presencia del síndrome preeclampsia - eclampsia. La adecuada placentación es la base para la tolerancia de la mujer al aloinjerto que es el embarazo, y se ve facilitada por los procesos inflamatorios que aseguran una 


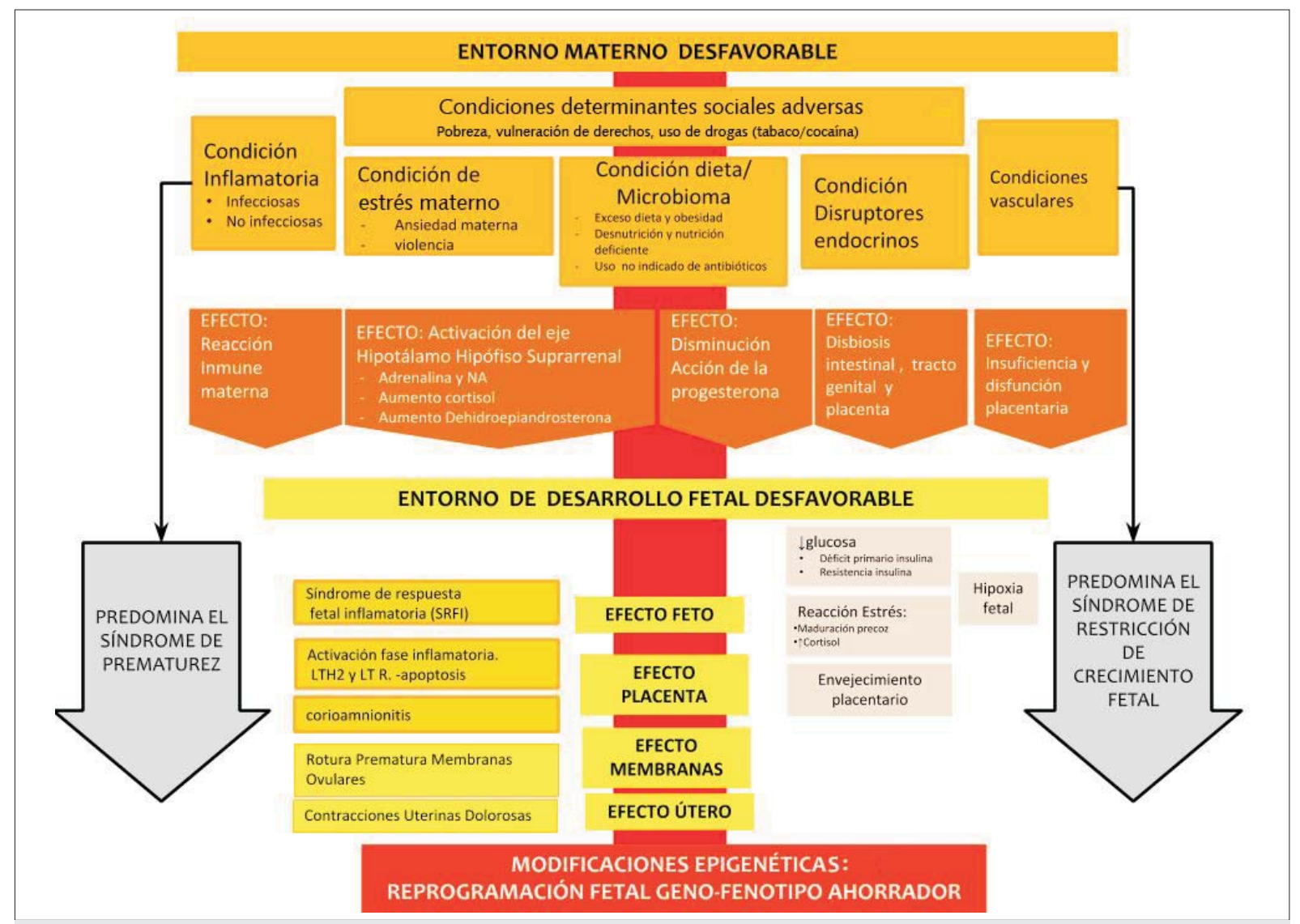

\section{Figura 1.}

adecuada remodelación vascular e invasión placentaria ${ }^{(9)}$. Luego de la fecundación y durante el primer trimestre, a nivel de la interfase materno-fetal, existe un predominio de expresión de citoquinas de perfil Th2 versus Th1, favorecido, entre otras circunstancias, por la acción de las hormonas sexuales, fundamentalmente la progesterona ${ }^{(4)}$. Esto asegura una disminución de la respuesta inmune local, lo que permite la adecuada remodelación de las arterias espiraladas uterinas por parte del trofoblasto. La placentación defectuosa es consecuencia de una respuesta inmune inadecuada en la cual los mecanismos inmunológicos locales se encuentran desbalanceados, lo que determina una pérdida de esa inmunotolerancia que no permite la correcta invasión trofoblástica. Se genera un ambiente de mal perfusión que determina un estrés oxidativo a nivel del lecho placentario, lo que conduce a una supresión selectiva de la síntesis de proteínas y una reducción de la proliferación celular. Esto puede verse agravado en caso de infartos placentarios y depósito de fibrina. En consecuencia, hay una reducción en el volumen de las vellosidades y el área de superficie para el in- tercambio materno-fetal. Se produce una desregulación extensa de la expresión génica, que afecta el transporte placentario, las funciones endocrina, metabólica e inmune ${ }^{(10)}$.

Cualesquiera sean estas condiciones adversas, el entorno materno desfavorable está vinculado estrechamente a los determinantes sociales perjudiciales. La pobreza y la exclusión son reconocidos universalmente por el impacto en las mujeres, sobre todo cuando hay intersección de vulnerabilidades como en lo etario (niñas, adolescentes y jóvenes), étnico racial (pueblos originarios y afrodescendientes), personas que viven crisis humanitarias y vinculadas con el cambio climático.

La condición materna desfavorable, por diferentes mecanismos, impacta en el entorno útero-placento-fetal, haciendo que este también sea desfavorable para el desarrollo del embarazo. Dentro de estos mecanismos se describen la respuesta inmune materna, como respuestas inflamatorias infecciosas y no infecciosas llevan a una inmunomodulación no acorde a la esperada para un embarazo normal ${ }^{(4)}$, la activación del eje hipotálamo hipófiso suprarrenal con aumento de la secreción de adre- 
nalina y noradrenalina, aumento de la actividad del cortisol, aumento de la dehidroepiandrosterona y aumento de la secreción y acción de la oxitocina ${ }^{(11)}$. También se describe la disminución de la acción de la progesterona, probablemente mediada por una serie concertada de eventos bioquímicos que afectan negativamente la capacidad del receptor de progesterona para regular genes diana que mantienen la quiescencia miometrial ${ }^{(12)}$. La disbiosis intestinal originada por los cambios en la microbiota, por un incremento de patógenos oportunistas y especies pro inflamatorias, puede desencadenar también un ambiente fetal desfavorable. La placentación defectuosa genera un intercambio materno-fetal inadecuado y un ambiente hipóxico local; estas alteraciones en la placentación se asocian tanto al PP como al RCIU ${ }^{(10)}$. Específicamente para el caso de la RCIU, puede presentarse aislado o asociado al síndrome de preeclampsia-eclampsia, en este último caso se asocia una disfunción placentaria más severa y mayor grado de vasculopatía materna ${ }^{(13)}$.

En función de la afectación que prime se puede generar un pretérmino o una RCIU. El síndrome de PP se produce cuando lo que prima es bien una respuesta a una respuesta inflamatoria, bien una infección como la corioamnionitis, bien la activación temprana de la fase inflamatoria de LTH2 y LTR, bien por la rotura de membranas, bien por hiperactividad uterina o bien por cualquier combinación de las anteriores. Por su parte, el RCIU se genera cuando predomina el déficit del metabolismo glucídico, la hipoxia y el estrés fetal. A pesar de la diferente presentación clínica en ambos síndromes se generan modificaciones epigenéticas, con activación de algunos genes y represión de otros, que dan como resultado el denominado geno-fenotipo ahorrador. Los mecanismos involucrados, la modificación de histonas, las micro-ARN, y la metilación de las citocinas, van a afectar al feto en desarrollo; por ejemplo, con alteración estructural de múltiples órganos y sistemas mayores que puede llevar a las afecciones de grado incremental donde se entrelazan la prematurez con la restricción del crecimiento fetal.

Dentro de la modificación epigenética, por ejemplo la salud mental, como la percepción de estrés, se relaciona con cambios epigenético fetales a través de células sanguíneas del cordón ${ }^{(14)}$. Otros factores estudiados han sido la violencia doméstica, con evidencia de su impacto sobre la metilación del gen del receptor glucocorticoide, y el bajo nivel socioeconómico, con la metilación de elementos que pueden ser utilizados como marcadores $^{(15,16)}$. Se ha descrito que las madres que sufren depresión durante el embarazo provocan un aumento en la metilación de genes específicos, provocando bajo peso al nacer y de ellos una disminución en la expresión del insulin growth factor 2 en comparación con los neonatos con peso adecuado ${ }^{(17)}$. La malnutrición es uno de los factores de riesgo que más se relaciona con el PP y el RCIU, y su asociación también ha quedado demostrada por cambios epigenéticos como en los estudios de hambrunas durante la Segunda Guerra Mundial ${ }^{(18)}$. Las disbiosis tienen un rol principal en la activación epigenética y para muchos autores sería el intermediario entre el ambiente y las condiciones psicosociales con los procesos que llevan a activar el genotipo ahorrador. Se desarrolla incluso una nueva disciplina, la nutrigenómica, que se enfoca en la relación entre nutrición, disbiosis y epigenética. La modulación epigenética, a nivel principalmente de las histonas, depende de los niveles de metabolitos intermediarios, vitaminas y minerales, los que actúan como sustratos, activadores o inhibidores, así la disbiosis tiene consecuencias directas en expresión génica mediante cambios epigenéticos y desarrollo de enfermedades, sobre todo metabólicas e inmunes ${ }^{(19)}$, (figura 2).

En la prematurez se describen cambios específicos en la metilación del ADN fetal con diferentes factores socioambientales, incluso se ha demostrado la persistencia del riesgo de prematurez de forma transgeneracional en una familia, dada la persistencia de los cambios epigenéticos ${ }^{(20)}$. Por ejemplo, en un estudio de pacientes africanas se demostró en los partos pretérminos una sobreexpresión del gen del complejo mayor de histocompatibilidad clase 1 por hipometilación a dicho nivel $^{(21)}$. Toda esta evidencia sobre la modulación epigenética en el riesgo de prematurez ha llevado a la definición de un modelo teórico conocido como Preterm Behavioral Epigenetics (PBE) ${ }^{(22)}$.

En los casos de restricción de crecimiento intrauterino también se ha demostrado la relación con cambios epigenéticos, sobre todo en aquellos genes que modulan el sistema endocrino metabólico ${ }^{(23)}$. Recientemente se han publicado dos estudios que apoyan estos resultados. Un estudio de casos y controles publicado en 2017, que comparaba fetos con RCIU con respecto a los que presentaban crecimiento acorde, encontró una metilación diferentes entre estos grupos en 2.265 genes $^{(24)}$. Otro estudio, publicado en 2018, comparó muestras placentarias y encontró diferentes patrones de metilación entre RCIU y crecimiento acorde, principalmente en los sectores asociados a riesgo cardiovascular ${ }^{(25)}$.

\section{Prematurez y restricción del crecimiento fetal y su relación con las enfermedades crónicas del adulto}

Cuando los principales síndromes obstétricos se desarrollan en contextos de pobreza y exclusión social la modulación epigenética que se estableció en la vida fetal no se revertirá espontáneamente y al mantenerse los 


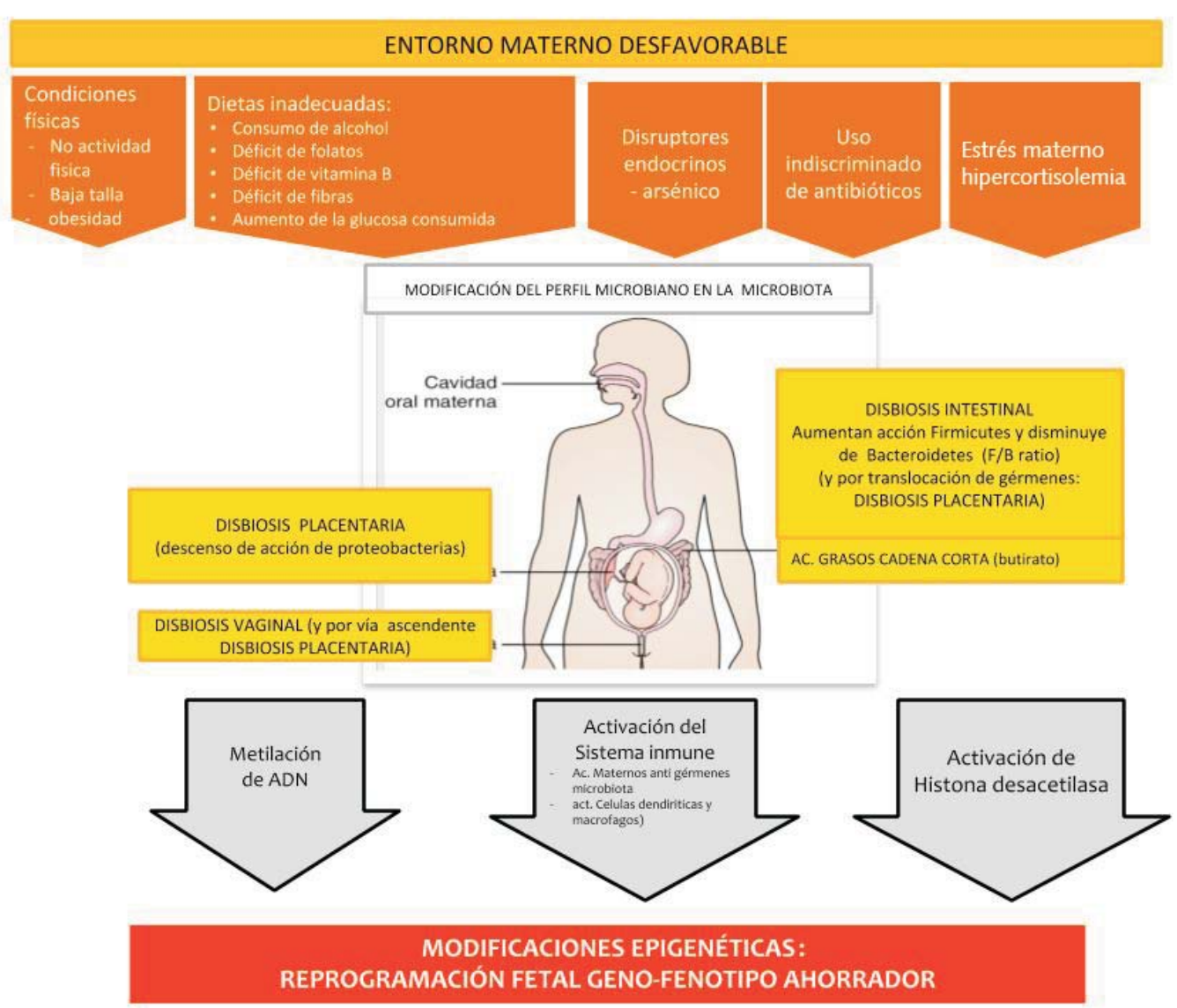

Figura 2.

contextos de pobreza y exclusión en la primera infancia con disfunciones en cuanto a crianza y alimentación, en el correr de la vida se verá un aumento de la prevalencia de anemia, obesidad y otras afecciones. En la juventud se consolidarán hábitos con sedentarismo, consumo de tóxicos y baja escolaridad que provoca un riesgo incrementado de desarrollo en la vida adulta de las enfermedades crónicas denominadas comúnmente enfermedades crónicas no transmisibles. Desde este razonamiento, en realidad, las enfermedades crónicas en los contextos de pobreza y exclusión sí son transmisibles, en este caso socialmente transmisibles ${ }^{(26)}$. Así entendidas, las enfermedades crónicas tendrían un fuerte vínculo de transmisión de una generación a la siguiente, no por herencia genética sino por impronta epigenética. Esta situación genera la hipótesis de la transmisión transgeneracional de las enfermedades crónicas en los contextos de pobreza y vulneración de derechos ${ }^{(27)}$, como se muestra en la figura 3.

Afortunadamente, la activación epigenética es reversible con la oportunidad de actuar revirtiendo los riesgos de enfermedades crónicas en el futuro. Este co- nocimiento científico es el que explica y justifica las políticas con discriminación positiva en el embarazo y en la primera infancia para revertir el impacto de las enfermedades crónicas.

\section{Conclusiones}

Los cambios epigenéticos podrían ser uno de los mecanismos más relevantes para plantear una perspectiva integral en el abordaje de los dos principales síndromes obstétricos. En particular, en el RCIU se podría incluso reconceptualizar como un estadio intermedio entre la fecundación y el desarrollo de enfermedades crónicas del adulto. Este abordaje promoverá la prevención específica con análisis de biomarcadores de activación del genotipo ahorrador, diagnóstico de tipo de activación epigenética y de disbiosis relacionadas, tratamiento específico en base a dietas que modifiquen las disbiosis o fármacos que reviertan la metilación. De avanzar en la investigación de biomarcadores y paneles de disbiosis específicos se podría incluso proponer nuevos enfoques de la conducta obstétrica que llevaran a interrumpir el embarazo una vez diagnosticados ambos síndromes, to- 


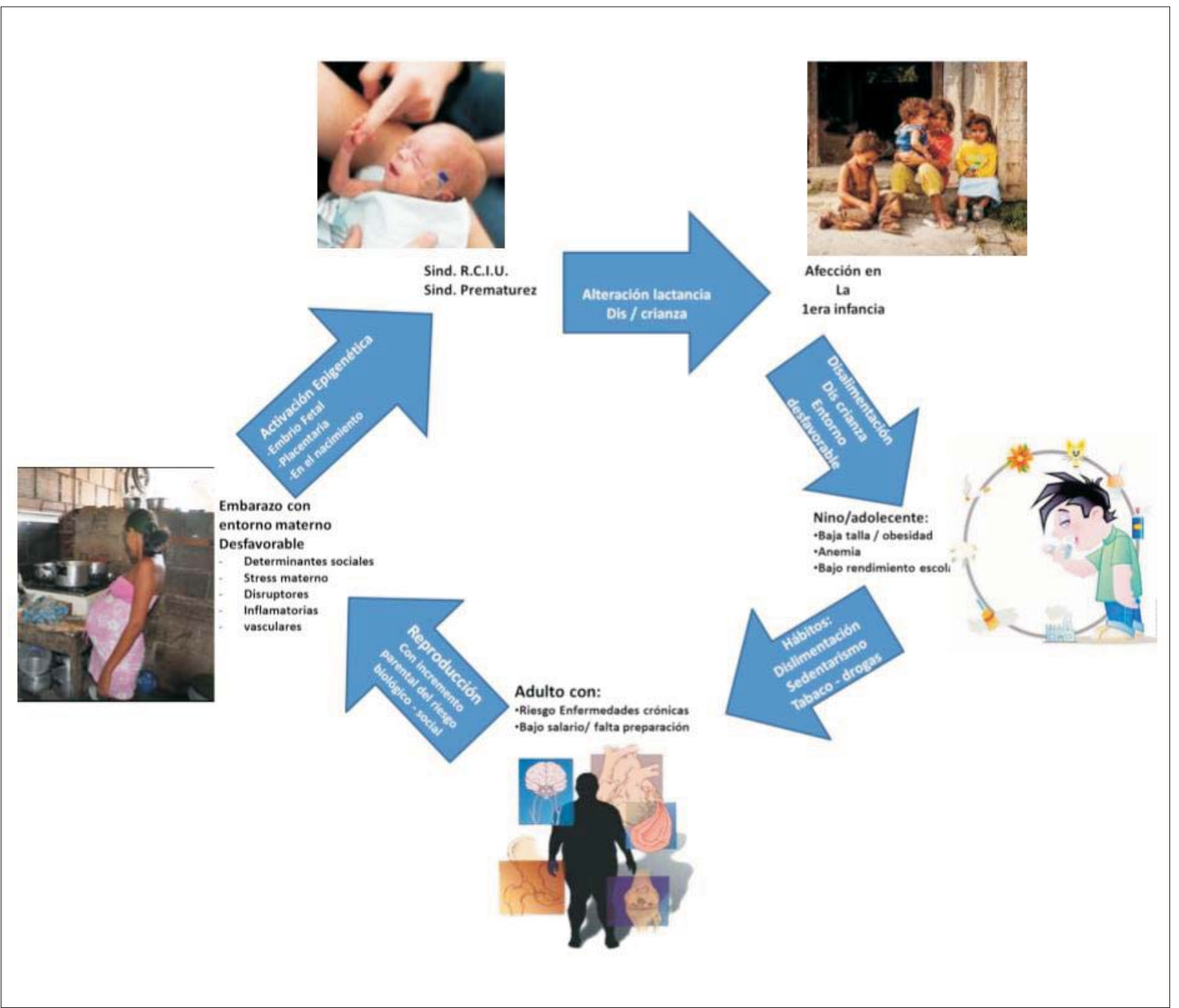

Figura 3.

mando en cuenta esta visión integral y no solo la posibilidad de muerte por asfixia en el caso del RCIU, o la consolidación de la corioamnionitis en los casos de PP vinculados, por ejemplo, a la rotura prematura y prolongada de membranas ovulares.

Por otra parte, conceptualizar que el punto de partida de las enfermedades crónicas se da durante la vida fetal es clave para el diseño e implementación de políticas públicas con discriminación positiva que vayan más allá de la primera infancia, abarcando el período de la gestación como prioridad, ya que es allí donde la modulación epigenética es mayor. Estas políticas tienen que armonizar los derechos de niños, niñas y adolescentes con los derechos de las mujeres, en particular los derechos sexuales y reproductivos, como garantía de integralidad en el abordaje. Por último, al entender que nuestro país crece demográficamente en base a la población más vulnerada en sus derechos, se comprenderá la prioridad absoluta de esta temática para la sostenibilidad de Uruguay como proyecto país.

\section{Abstract}

Preterm birth and growth restriction are obstetric syndromes which share etiopathogenic and pathophysiological mechanisms that often interact and feed from each other. Etiologically, they may be classified into inflammation, maternal stress, low socio-economic background and vulnerability of rights, endocrine disruptors, diet and microbiota alterations and vascular conditions, depending on specific conditions. These conditions, either in isolation or more often combined, create an unfavorable environment for the development of pregnancy, causing specific effects such as maternal immune response that may be mediated by infections, the activation of the hypothalamic-pituitary-adre- 
nal (HPA) axis, drop in progesterone levels, dysbiosis, both intestinal and vaginal, and placental dysfunction caused by ageing. The unfavorable environment has an impact on the utero-feto-placental unit resulting in either preterm birth of growth restriction, depending on the predominance of the different responses. Regardless of the prevailing syndromic response, both preterm birth and growth restriction share the development of the thrifty pheno-genotype, essential for fetal survival. The cost of this epigenetic modulation is an increase in chronic adult diseases, which, conceptually, are transmissible diseases due to social vulnerability where these people live.

\section{Resumo}

As síndromes obstétricas de parto de pré-termo (PP) e de restrição do crescimento fetal (RCIU) compartem mecanismos etiopatogênicos e fisiopatológicos, que muitas vezes interagem e se retroalimentam. Do ponto de vista da etiologia, as condições específicas que as geram podem ser classificadas esquematicamente em inflamação, estresse materno, déficit nas condições socio econômicas e vulnerabilidade de direitos, ação de disruptores endócrinos, alterações da dieta e da microbiota e afecções vasculares. Estas condições, agindo de forma isolada ou mais frequentemente combinada, geram um ambiente materno desfavorável para o desenvolvimento da gravidez provocando efeitos específicos como a reação imune materna, mediada ou não pela presença de infecções, a ativação do eixo hipotálamo - hipófiso - adrenal, a diminuição da ação da progesterona, as disbioses, tanto intestinais como vaginais e a disfunção por envelhecimento placentário. Esse ambiente desfavorável impactará na unidade útero-placento-fetal, produzindo PP ou RCIU, de acordo com a preferência de diferentes respostas. Independentemente de qual das respostas sindromáticas predomine, ambos PP e RCIU, têm em comum o desenvolvimento do feno-genótipo poupador, imprescindível para a sobrevida fetal. $O$ custo desta modulação epigenética é o aumento das doenças crônicas do adulto, que conceitualmente, são patologias transmissíveis pela vulnerabilidade social na qual o ciclo de vida dessas pessoas se desenvolve.

\section{Bibliografía}

1. Blencowe H, Cousens S, Oestergaard M, Chou D, Moller A, Narwal R, et al. National, regional, and worldwide estimates of preterm birth rates in the year 2010 with time trends since 1990 for selected countries: a systematic analysis and implications. Lancet 2012; 379(9832):2162-72.

2. Lee A, Kozuki N, Cousens S, Stevens G, Blencowe H, Silveira $\mathbf{M}$, et al. Estimates of burden and consequences of infants born small for gestational age in low and middle income countries with INTERGROWTH-21 st standard: analysis of CHERG datasets. BMJ 2017; 358:j3677.

3. Drake A, Walker B. The intergenerational effects of fetal programming: non-genomic mechanisms for the inheritance of low birth weight and cardiovascular risk. J Endocrinol 2004; 180(1):1-16.

4. Sykes L, MacIntyre D, Yap X, Teoh T, Bennett P. The Th1:th2 dichotomy of pregnancy and preterm labour. Mediators Inflamm 2012; 2012:967629.

5. Hill A, Pallitto C, McCleary-Sills J, Garcia-Moreno C. A systematic review and meta-analysis of intimate partner violence during pregnancy and selected birth outcomes. Int $\mathrm{J}$ Gynaecol Obstet 2016; 133(3):269-76.

6. Stanislawski M, Dabelea D, Wagner B, Sontag M, Lozupone C, Eggesbø M. Pre-pregnancy weight, gestational weight gain, and the gut microbiota of mothers and their infants. Microbiome 2017; 5(1):113.

7. Ferguson K, McElrath T, Chen Y, Mukherjee B, Meeker J. Urinary phthalate metabolites and biomarkers of oxidative stress in pregnant women: a repeated measures analysis. Environ Health Perspect 2015; 123(3):210-6.

8. Porter T, Kent S, Su W, Beck H, Gohlke J. Spatiotemporal association between birth outcomes and coke production and steel making facilities in Alabama, USA: a cross-sectional study. Environ Health 2014; 13:85.

9. Burton G, Fowden A, Thornburg K. Placental origins of chronic disease. Physiol Rev 2016; 96(4):1509-65.

10. Burton G, Jauniaux E. Pathophysiology of placental-derived fetal growth restriction. Am J Obstet Gynecol 2018; 218(2S):S745-61.

11. Kajantie E. Fetal origins of stress-related adult disease. Ann N Y Acad Sci 2006; 1083:11-27.

12. Hardy D, Janowski B, Corey D, Mendelson C. Progesterone receptor plays a major antiinflammatory role in human myometrial cells by antagonism of nuclear factor-kappaB activation of cyclooxygenase 2 expression. Mol Endocrinol 2006; 20(11):2724-33.

13. Maršál K. Preeclampsia and intrauterine growth restriction: placental disorders still not fully understood. J Perinat Med 2017; 45(7):775-777.

14. Vidal A, Benjamin Neelon S, Liu Y, Tuli A, Fuemmeler B, Hoyo C, et al. Maternal stress, preterm birth, and DNA methylation at imprint regulatory sequences in humans. Genet Epigenet 2014; 6:37-44.

15. Radtke K, Ruf M, Gunter H, Dohrmann K, Schauer M, Meyer A, et al. Transgenerational impact of intimate partner violence on methylation in the promoter of the glucocorticoid receptor. Transl Psychiatry 2011; 1(7):e21.

16. Tehranifar P, Wu H, Fan X, Flom J, Ferris J, Cho Y, et al. Early life socioeconomic factors and genomic DNA methylation in mid-life. Epigenetics 2013; 8(1):23-7.

17. Liu Y, Murphy S, Murtha A, Fuemmeler B, Schildkraut J, Huang $\mathbf{Z}$, et al. Depression in pregnancy, infant birth weight and DNA methylation of imprint regulatory elements. Epigenetics 2012; 7(7):735-46. 
18. Tobi E, Lumey L, Talens R, Kremer D, Putter H, Stein A, et al. DNA methylation differences after exposure to prenatal famine are common and timing- and sex-specific. Hum Mol Genet 2009; 18(21):4046-53.

19. Krautkramer K, Dhillon R, Denu J, Carey H. Metabolic programming of the epigenome: host and gut microbial metabolite interactions with host chromatin. Transl Res 2017; 189:30-50

20. Burris H, Baccarelli A, Wright R, Wright R. Epigenetics: linking social and environmental exposures to preterm birth. Pediatr Res 2016; 79(1-2):136-40.

21. Parets S, Conneely K, Kilaru V, Menon R, Smith A. DNA methylation provides insight into intergenerational risk for preterm birth in African Americans. Epigenetics 2015; 10(9):784-92.

22. Montirosso R, Casini E, Provenzi L, Putnam S, Morandi F, Fedeli C, et al. A categorical approach to infants' individual differences during the Still-Face paradigm. Infant Behav Dev 2015; 38:67-76.
23. Banister C, Koestler D, Maccani M, Padbury J, Houseman E, Marsit C. Infant growth restriction is associated with distinct patterns of DNA methylation in human placentas. Epigenetics 2011; 6(7):920-7.

24. Ding Y, Cui H. Integrated analysis of genome-wide DNA methylation and gene expression data provide a regulatory network in intrauterine growth restriction. Life Sci 2017; 179:60-5.

25. Chen P, Chu A, Liao W, Rubbi L, Janzen C, Hsu F, et al. Prenatal growth patterns and birthweight are associated with differential dna methylation and gene expression of cardiometabolic risk genes in human placentas: a discovery-based approach. Reprod Sci 2018; 25(4):523-39.

26. Barker D. The developmental origins of chronic adult disease. Acta Paediatr Suppl 2004; 93(446):26-33.

27. Briozzo L, Coppola F, Gesuele J, Tomasso G. Restricción de crecimiento fetal, epigenética y transmisión trans generacional de las enfermedades crónicas y la pobreza. Horiz Med $2013 ; 13(4): 45-53$.

\section{Contribución de autores}

Los autores participaron de todas las etapas (concepción, diseño, recolección de datos, análisis redacción y revisión) en igual medida.

Leonel Briozzo, https://orcid.org/0000-0002-6772-3188

Stephanie Viroga, https://orcid.org/0000-0001-9065-6692 